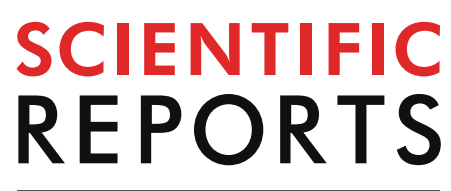

natureresearch

Check for updates

\title{
Relative overhydration
} is independently associated with left ventricular hypertrophy in dialysis naïve patients with stage 5 chronic kidney disease

\begin{abstract}
Byoung-Geun Han, Jun Young Lee, Seung Ok Choi, Jae-Won Yang \& Jae-Seok Kim ${ }^{\bowtie}$
Patients with chronic kidney disease (CKD) have a high prevalence of left ventricular hypertrophy (LVH), which increases as kidney function decreases. LVH pathophysiology is complex, making it difficult to generalise its evolution in CKD. Therefore, early detection and prevention of risk factors are critical. Assessment and management of volume status can minimise cardiovascular complications including LVH. We retrospectively investigated the associations between fluid overload and LVH in patients with stage 5 CKD not undergoing dialysis in prospective cohort of 205 patients (age: $59.34 \pm 13.51$ years; women: $43.4 \%$ ). All patients, free of intrinsic heart disease, were assessed for relative overhydration/extracellular water $(\mathrm{OH} / \mathrm{ECW})$ by bioimpedance spectroscopy. Our results show that markers reflecting fluid balance were significantly higher in the LVH group and as $\mathrm{OH}$ / ECW increased, the left ventricular mass index (LVMI) trended higher. Furthermore, our results show that systolic blood pressure, serum phosphorus levels, and $\mathrm{OH} / \mathrm{ECW}$ were independently associated with LVMI and that $\mathrm{OH} / \mathrm{ECW}$ was independently associated with LVH. Structural and functional evaluation of the heart using echocardiography and volume status assessment using bioimpedance should be performed simultaneously in patients with early-stage CKD, even in those without evident cardiovascular disease.
\end{abstract}

Cardiovascular complications are the major cause of mortality and morbidity in patients with end-stage renal disease (ESRD). Structural and functional cardiac changes, such as left ventricular hypertrophy (LVH) and left ventricular (LV) diastolic dysfunction, can be seen in patients with even mild-to-moderate chronic kidney disease (CKD) regardless of the cause. Patients with CKD have a high prevalence of LVH, ranging from 29 to $74 \%$ in different studies, and its prevalence increases as kidney function decreases ${ }^{1-7}$.

However, the pathophysiology of LVH has not been completely elucidated in patients with gradually decreasing renal function due to the complexity of traditional and non-traditional cardiovascular risk factors. The clinical course and patterns of LVH are also determined by the stage of renal dysfunction. Previous studies regarding LVH have focused on patients with CKD who have a wide range of kidney function or patients with ESRD undergoing dialysis ${ }^{8-13}$.

The assessment and management of volume status are especially important as it can promote hemodynamic stability, minimise cardiovascular complications including $\mathrm{LVH}$, and reduce the risk of cardiovascular events in patients with $\mathrm{CKD}^{14}$. $\mathrm{LVH}$ is a strong predictor of the risk of poor cardiovascular and renal outcomes ${ }^{15-17}$. Since LVH has been observed even in early-stage CKD and was associated with fluid overload, an early therapeutic approach to correct volume status has been suggested ${ }^{18}$. Bioimpedance is a non-invasive, quick, and relatively affordable method to quantitatively assess the volume status of a patient, although only a small number of studies have examined the relationship between volume status and $\mathrm{LVH}^{11,12,19}$.

An analysis regarding estimated glomerular filtration rate (eGFR) in a homogenous population is required. Furthermore, a sensitive therapeutic marker indicating precisely how much fluid overload is associated with 
A total of 276 patients were enrolled in the bioimpedance cohort

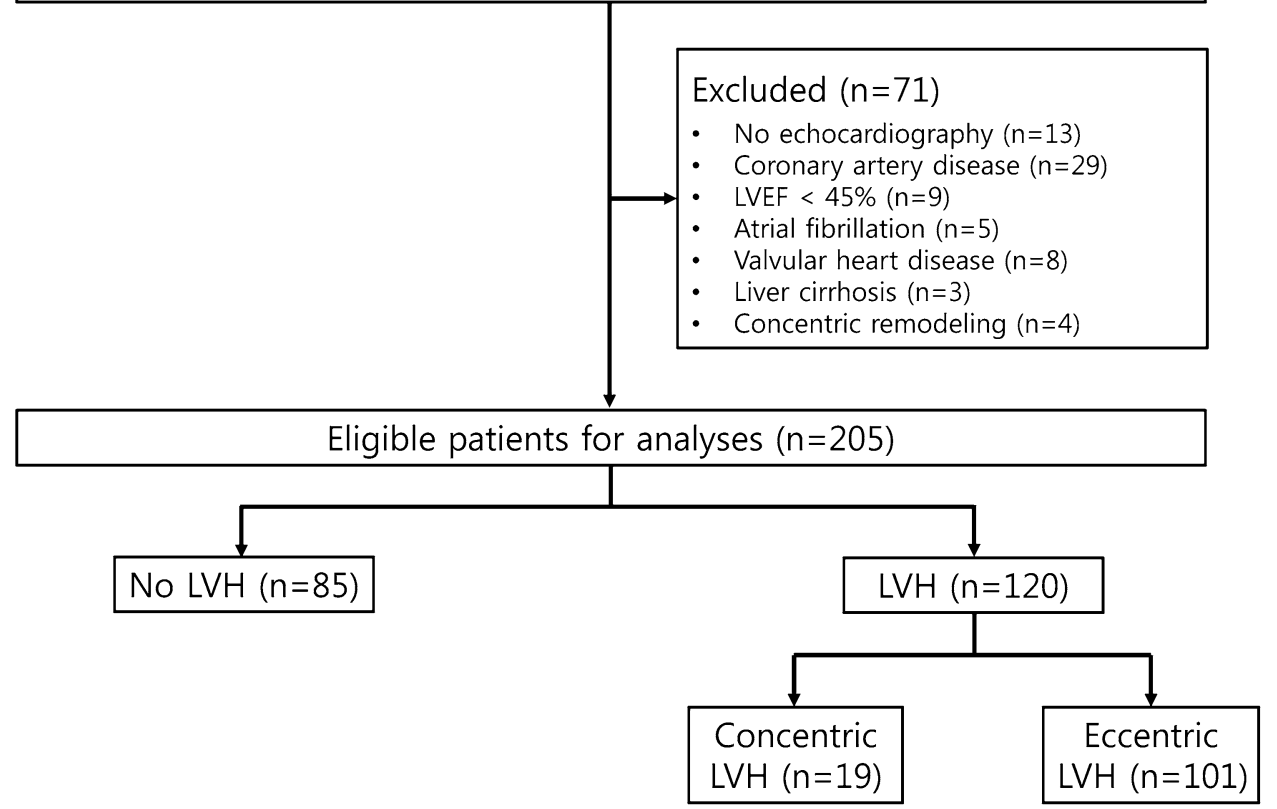

Figure 1. Flow diagram of patient selection in this study. $L V E F$ left ventricular ejection fraction, $L V H$ left ventricular hypertrophy.

LVH risk is required. Therefore, in this study, we particularly focused on the relationship between actual fluid overload and LVH in patients with stage $5 \mathrm{CKD}$ not undergoing dialysis (CKD5-ND).

\section{Results}

Characteristics of the study patients. Figure 1 depicts the flow diagram of the study. After the application of the exclusion criteria, a total of 205 patients (mean age, $59.34 \pm 13.51$ years; $43.4 \%$ females) were analysed. The clinical characteristics of each group according to the absence or presence of LVH are presented in Table 1. Among the total patients, $62.0 \%$ were aged below 65 years and $63.4 \%$ had diabetes. On echocardiography, a total of 120 patients (58.5\%) had LVH (LVH group). LVH was more prevalent in women than in men (74.2 vs. 46.6\%; $P=0.001)$. The systolic blood pressure (SBP) was significantly higher in the LVH group $(P=0.010)$. Among the echocardiographic parameters, the left atrial (LA) dimension (LAD), LA volume index (LAVI), E/e' ratio, LV end-diastolic dimension (LVEDD), LV end-diastolic volume (LVEDV), left ventricular mass index (LVMI), and relative wall thickness (RWT) were substantially greater in the LVH group. Patients with LVH had lower levels of eGFR, albumin, calcium, and haemoglobin, but had considerably higher phosphorus levels. The levels of high-sensitivity C-reactive protein (hs-CRP) and intact parathyroid hormone (iPTH) did not differ between the two groups. Markers reflecting fluid balance such as overhydration $(\mathrm{OH}), \mathrm{OH} /$ extracellular water (ECW), ECW/total body water (TBW), and N-terminal prohormone of B-type natriuretic peptide (NT-proBNP) were substantially higher in the LVH group.

The clinical characteristics of the patients according to the tertiles of OH/ECW are presented in Table 2. Compared with patients in the first tertile of $\mathrm{OH} / \mathrm{ECW}$, patients in the third tertile were more likely to have a history of diabetes, have a higher SBP, and have been treated with diuretics. There was a trend of higher levels of serum phosphorus, hs-CRP, and NT-proBNP across increasing tertiles of OH/ECW. In contrast, eGFR, haemoglobin, albumin, and calcium levels were lower in the third tertile group than in the first tertile group. Aggravation of the $\mathrm{OH} / \mathrm{ECW}$ was substantially associated with echocardiographic findings, including LAD, LAVI, E/e' ratio, LVEDD, LVEDV, LVMI, and RWT, while LV ejection fraction (LVEF) showed no difference between the three groups (Fig. 2).

As assessed by RWT and LVMI, the prevalences of eccentric LVH (eLVH) were $37.3 \%, 49.3 \%$, and $61.2 \%$ and those of concentric LVH (cLVH) were $4.5 \%, 10.4 \%$, and $11.9 \%$ in tertiles 1,2 , and 3 of OH/ECW, respectively, suggesting that eLVH progressively increased across increasing tertiles of OH/ECW $(P$ for trend $<0.001)$ (Fig. 3$)$.

Multivariate analyses. In stepwise multiple linear regressions analyses, OH/ECW, SBP, and serum phosphorus were shown to be substantially associated with LVMI after adjustment for clinical confounding factors including age, prevalent diabetes, diuretics use, hs-CRP, albumin, and eGFR, while sex, body mass index (BMI), haemoglobin, serum calcium, and SBP were significantly associated with LVEDD (Table 3).

When evaluating $\mathrm{OH} / \mathrm{ECW}$ as a continuous variable, the association of OH/ECW with LVMI remained statistically significant. When evaluating $\mathrm{OH} / \mathrm{ECW}$ as a categorical variable for further analysis, the substantial association between OH/ECW and LVMI disappeared in model 3, as did the other two variables (Table 4). 


\begin{tabular}{|c|c|c|c|c|}
\hline Variables & Total $(n=205)$ & No LVH $(n=85)$ & LVH $(n=120)$ & $P$ value \\
\hline Age, years & $59.34 \pm 13.51$ & $58.52 \pm 13.54$ & $59.92 \pm 13.52$ & 0.466 \\
\hline \multicolumn{5}{|l|}{ Age } \\
\hline$<65$ years & $127(62.0 \%)$ & $55(43.3 \%)$ & $72(56.7 \%)$ & 0.494 \\
\hline$\geq 65$ years & $78(38.0 \%)$ & $30(38.5 \%)$ & $48(61.5 \%)$ & \\
\hline \multicolumn{5}{|l|}{ Sex } \\
\hline Male & $116(56.6 \%)$ & $62(53.4 \%)$ & $54(46.6 \%)$ & 0.001 \\
\hline Female & $89(43.4 \%)$ & $23(25.8 \%)$ & $66(74.2 \%)$ & \\
\hline \multicolumn{5}{|l|}{ Diabetes } \\
\hline Yes & $130(63.4 \%)$ & $51(39.2 \%)$ & $79(60.8 \%)$ & 0.393 \\
\hline No & $75(36.6 \%)$ & $34(45.3 \%)$ & $41(54.7 \%)$ & \\
\hline \multicolumn{5}{|l|}{ Diuretics } \\
\hline Yes & $130(63.4 \%)$ & $52(61.2 \%)$ & $78(65.0 \%)$ & 0.576 \\
\hline No & $75(36.6 \%)$ & $33(38.8 \%)$ & $42(35.0 \%)$ & \\
\hline \multicolumn{5}{|l|}{ RAASi } \\
\hline Yes & $145(70.7 \%)$ & $58(68.2 \%)$ & $87(72.5 \%)$ & 0.509 \\
\hline No & $60(29.3 \%)$ & $27(31.8 \%)$ & $33(27.5 \%)$ & \\
\hline \multicolumn{5}{|l|}{ Phosphorous binders } \\
\hline Yes & $31(15.1 \%)$ & $11(12.9 \%)$ & $20(16.7 \%)$ & 0.463 \\
\hline No & $174(84.9 \%)$ & $74(87.1 \%)$ & $100(83.3 \%)$ & \\
\hline SBP, mmHg & $142.19 \pm 19.11$ & $138.09 \pm 20.08$ & $145.08 \pm 17.92$ & 0.010 \\
\hline $\mathrm{DBP}, \mathrm{mmHg}$ & $80.42 \pm 11.21$ & $81.29 \pm 12.22$ & $79.80 \pm 10.45$ & 0.348 \\
\hline BMI, $\mathrm{kg} / \mathrm{m}^{2}$ & $25.05 \pm 4.12$ & $24.89 \pm 4.06$ & $25.17 \pm 4.18$ & 0.635 \\
\hline LAD, cm & $4.60 \pm 0.46$ & $4.45 \pm 0.47$ & $4.70 \pm 0.43$ & $<0.001$ \\
\hline LAVI, $\mathrm{mL} / \mathrm{m}^{2}$ & $37.22 \pm 10.05$ & $32.11 \pm 7.37$ & $40.84 \pm 10.14$ & $<0.001$ \\
\hline $\mathrm{E} / \mathrm{e}^{\prime}$ ratio & $15.32 \pm 5.29$ & $13.36 \pm 3.94$ & $16.71 \pm 5.69$ & $<0.001$ \\
\hline LVEDD, cm & $5.40 \pm 0.46$ & $5.27 \pm 0.41$ & $5.49 \pm 0.47$ & $<0.001$ \\
\hline LVEDV, mL & $142.96 \pm 28.35$ & $134.61 \pm 24.08$ & $148.00 \pm 29.73$ & $<0.001$ \\
\hline LVMI, $\mathrm{g} / \mathrm{m}^{2}$ & $114.72 \pm 25.14$ & $93.86 \pm 13.29$ & $129.50 \pm 20.67$ & $<0.001$ \\
\hline RWT & $0.35 \pm 0.06$ & $0.33 \pm 0.04$ & $0.37 \pm 0.06$ & $<0.001$ \\
\hline LVEF, \% & $63.34 \pm 5.56$ & $63.00 \pm 5.11$ & $63.58 \pm 5.86$ & 0.467 \\
\hline NT-proBNP, pg/mL ${ }^{\mathrm{a}}$ & $2,334(657-8,014)$ & $840(351-5,803)$ & $3,530(1,329-10,102)$ & $<0.001$ \\
\hline hs-CRP, mg/dL & $1.31 \pm 2.95$ & $1.47 \pm 2.91$ & $1.19 \pm 2.98$ & 0.513 \\
\hline iPTH, pg/mL & $301.18 \pm 201.87$ & $296.33 \pm 227.71$ & $304.65 \pm 182.12$ & 0.772 \\
\hline Haemoglobin, g/dL & $9.04 \pm 1.26$ & $9.28 \pm 1.24$ & $8.87 \pm 1.24$ & 0.020 \\
\hline Total protein, g/dL & $6.15 \pm 0.77$ & $6.36 \pm 0.81$ & $6.00 \pm 0.70$ & 0.001 \\
\hline Albumin, g/dL & $3.49 \pm 0.55$ & $3.63 \pm 0.53$ & $3.39 \pm 0.55$ & 0.002 \\
\hline Total cholesterol, mg/dL & $146.69 \pm 40.12$ & $140.91 \pm 41.11$ & $150.82 \pm 39.05$ & 0.082 \\
\hline $\mathrm{HDL}-\mathrm{C}, \mathrm{mg} / \mathrm{dL}$ & $38.34 \pm 12.77$ & $37.04 \pm 12.74$ & $39.27 \pm 12.76$ & 0.227 \\
\hline LDL-C, mg/dL & $81.62 \pm 35.30$ & $76.22 \pm 35.33$ & $85.47 \pm 34.93$ & 0.070 \\
\hline Triglyceride, mg/dL & $132.41 \pm 72.70$ & $139.16 \pm 74.24$ & $127.59 \pm 71.50$ & 0.263 \\
\hline Calcium, mg/dL & $7.75 \pm 1.06$ & $7.95 \pm 1.10$ & $7.61 \pm 1.00$ & 0.023 \\
\hline Phosphorus, mg/dL & $6.01 \pm 1.54$ & $5.61 \pm 1.52$ & $6.30 \pm 1.49$ & 0.001 \\
\hline $\mathrm{eGFR}, \mathrm{mL} / \mathrm{min} / 1.73 \mathrm{~m}^{2}$ & $6.80 \pm 2.46$ & $7.58 \pm 2.72$ & $6.25 \pm 2.10$ & $<0.001$ \\
\hline $\mathrm{OH}$, litre & $2.89 \pm 3.23$ & $2.05 \pm 2.85$ & $3.49 \pm 3.36$ & 0.002 \\
\hline $\mathrm{OH} / \mathrm{ECW}, \%$ & $15.02 \pm 14.65$ & $10.72 \pm 14.11$ & $18.11 \pm 14.30$ & $<0.001$ \\
\hline ECW/TBW & $0.50 \pm 0.05$ & $0.49 \pm 0.04$ & $0.51 \pm 0.05$ & $<0.001$ \\
\hline
\end{tabular}

Table 1. Comparison of demographics, serum chemistry, echocardiographic findings, and volume status between patients with and without left ventricular hypertrophy. BMI body mass index, DBP diastolic blood pressure, $E C W$ extracellular water, $e G F R$ estimated glomerular filtration rate, $H D L-C$ high-density lipoprotein cholesterol, $h s-C R P$ high-sensitivity C-reactive protein, $i P T H$ intact parathyroid hormone, $L A D$ left atrial dimension, $L A V I$ left atrial volume index, $L D L-C$ low-density lipoprotein cholesterol, $L V E D D$ left ventricular end-diastolic dimension, $L V E F$ left ventricular ejection fraction, $L V E D V$ left ventricular end-diastolic volume, $L V H$ left ventricular hypertrophy, $L V M I$ left ventricular mass index, NT-proBNP N-terminal prohormone of B-type natriuretic peptide, $O H$ overhydration, $R A A S i$ Renin-angiotensin-aldosterone system inhibitors, $R W T$ relative wall thickness, $S B P$ systolic blood pressure, $T B W$ total body water. ${ }^{a}$ Mann-Whitney $U$ test; Median (interquartile range). 


\begin{tabular}{|c|c|c|c|c|c|}
\hline \multirow[b]{2}{*}{ Variables } & \multicolumn{3}{|l|}{ OH/ECW (\%) } & \multirow[b]{2}{*}{$P$ value } & \multirow[b]{2}{*}{$P$ for trend ${ }^{*}$} \\
\hline & Tertile 1 & Tertile 2 & \begin{tabular}{|l|} 
Tertile 3 \\
\end{tabular} & & \\
\hline Age, years & $59.01 \pm 14.21$ & $60.91 \pm 12.10$ & $57.46 \pm 14.22$ & 0.339 & 0.383 \\
\hline \multicolumn{6}{|l|}{ Age } \\
\hline$<65$ years & $41(32.5 \%)$ & $40(31.7 \%)$ & $45(35.7 \%)$ & 0.640 & 0.476 \\
\hline$\geq 65$ years & $26(34.7 \%)$ & $27(36.0 \%)$ & $22(29.3 \%)$ & & \\
\hline \multicolumn{6}{|l|}{ Sex } \\
\hline Male & $31(27.2 \%)$ & $44(38.6 \%)$ & $39(34.2 \%)$ & 0.073 & 0.164 \\
\hline Female & $36(41.4 \%)$ & $23(26.4 \%)$ & $28(32.2 \%)$ & & \\
\hline \multicolumn{6}{|l|}{ Diabetes } \\
\hline Yes & $31(24.6 \%)$ & $42(33.3 \%)$ & $53(42.1 \%)$ & $<0.001$ & $<0.001$ \\
\hline No & $36(48.0 \%)$ & $25(33.3 \%)$ & $14(18.7 \%)$ & & \\
\hline \multicolumn{6}{|l|}{ Diuretics } \\
\hline Yes & $38(29.9 \%)$ & $38(29.9 \%)$ & $51(40.2 \%)$ & 0.027 & 0.020 \\
\hline No & $29(39.2 \%)$ & $29(39.2 \%)$ & $16(21.6 \%)$ & & \\
\hline \multicolumn{6}{|l|}{ RAASi } \\
\hline Yes & $48(34.0 \%)$ & $46(32.6 \%)$ & $47(33.3 \%)$ & 0.931 & 0.851 \\
\hline No & $19(31.7 \%)$ & $21(35.0 \%)$ & $20(33.3 \%)$ & & \\
\hline \multicolumn{6}{|l|}{ Phosphorous binders } \\
\hline Yes & $7(22.6 \%)$ & $9(29.0 \%)$ & $15(48.4 \%)$ & 0.138 & 0.056 \\
\hline No & $60(35.3 \%)$ & $58(34.1 \%)$ & $52(30.6 \%)$ & & \\
\hline SBP, mmHg & $134.18 \pm 18.27$ & $147.61 \pm 18.22$ & $145.01 \pm 17.96$ & $<0.001$ & 0.002 \\
\hline DBP, $\mathrm{mmHg}$ & $78.57 \pm 11.17$ & $82.06 \pm 11.77$ & $81.06 \pm 10.68$ & 0.181 & 0.245 \\
\hline BMI, $\mathrm{kg} / \mathrm{m}^{2}$ & $25.32 \pm 3.84$ & $24.70 \pm 3.98$ & $25.08 \pm 4.62$ & 0.691 & 0.489 \\
\hline LAD, cm & $4.43 \pm 0.42$ & $4.61 \pm 0.42$ & $4.73 \pm 0.49$ & 0.001 & $<0.001$ \\
\hline LAVI, $\mathrm{mL} / \mathrm{m}^{2}$ & $32.07 \pm 8.41$ & $37.57 \pm 8.15$ & $41.58 \pm 10.79$ & $<0.001$ & $<0.001$ \\
\hline $\mathrm{E} / \mathrm{e}^{\prime}$ ratio & $13.42 \pm 3.61$ & $15.03 \pm 5.17$ & $17.53 \pm 6.12$ & $<0.001$ & $<0.001$ \\
\hline LVEDD, cm & $5.27 \pm 0.40$ & $5.48 \pm 0.47$ & $5.45 \pm 0.48$ & 0.015 & 0.028 \\
\hline LVEDV, mL & $135.36 \pm 23.49$ & $147.78 \pm 29.60$ & $146.33 \pm 30.00$ & 0.020 & 0.034 \\
\hline LVMI, $\mathrm{g} / \mathrm{m}^{2}$ & $104.28 \pm 21.27$ & $116.57 \pm 23.01$ & $123.55 \pm 27.23$ & $<0.001$ & $<0.001$ \\
\hline RWT & $0.34 \pm 0.05$ & $0.35 \pm 0.06$ & $0.36 \pm 0.06$ & 0.023 & 0.001 \\
\hline LVEF, \% & $63.40 \pm 5.25$ & $62.78 \pm 5.36$ & $63.81 \pm 5.87$ & 0.552 & 0.933 \\
\hline NT-proBNP, pg/mL ${ }^{\mathrm{a}}$ & $582(318-1,665)$ & $1,959(748-5,077)$ & $8,182(4,350-20,855)$ & $<0.001$ & $<0.001$ \\
\hline hs-CRP, mg/dL & $0.69 \pm 2.00$ & $1.04 \pm 2.68$ & $2.13 \pm 3.73$ & 0.017 & $<0.001$ \\
\hline iPTH, pg/mL & $325.17 \pm 268.43$ & $308.97 \pm 173.62$ & $276.44 \pm 145.07$ & 0.372 & 0.616 \\
\hline Haemoglobin, g/dL & $9.55 \pm 1.20$ & $8.99 \pm 1.22$ & $8.64 \pm 1.14$ & $<0.001$ & $<0.001$ \\
\hline Total protein, g/dL & $6.66 \pm 0.63$ & $6.18 \pm 0.56$ & $5.59 \pm 0.71$ & $<0.001$ & $<0.001$ \\
\hline Albumin, g/dL & $3.85 \pm 0.39$ & $3.57 \pm 0.44$ & $3.03 \pm 0.47$ & $<0.001$ & $<0.001$ \\
\hline Total cholesterol, mg/dL & $153.21 \pm 40.01$ & $135.33 \pm 33.87$ & $150.94 \pm 44.46$ & 0.019 & 0.553 \\
\hline HDL-C, mg/dL & $38.65 \pm 11.69$ & $37.56 \pm 12.35$ & $38.83 \pm 14.46$ & 0.831 & 0.761 \\
\hline LDL-C, mg/dL & $83.19 \pm 36.05$ & $75.32 \pm 30.80$ & $86.28 \pm 38.82$ & 0.190 & 0.781 \\
\hline Triglyceride, $\mathrm{mg} / \mathrm{dL}$ & $153.69 \pm 97.62$ & $115.70 \pm 57.18$ & $125.91 \pm 50.22$ & 0.007 & 0.191 \\
\hline Calcium, mg/dL & $8.23 \pm 1.17$ & $7.69 \pm 0.91$ & $7.32 \pm 0.88$ & $<0.001$ & $<0.001$ \\
\hline Phosphorus, mg/dL & $5.48 \pm 1.29$ & $6.19 \pm 1.40$ & $6.36 \pm 1.75$ & 0.002 & 0.001 \\
\hline $\mathrm{eGFR}, \mathrm{mL} / \mathrm{min} / 1.73 \mathrm{~m}^{2}$ & $7.58 \pm 2.36$ & $6.65 \pm 2.51$ & $6.17 \pm 2.23$ & 0.003 & 0.001 \\
\hline $\mathrm{OH}$, litre & $0.04 \pm 0.89$ & $2.12 \pm 0.74$ & $6.51 \pm 2.84$ & $<0.001$ & $<0.001$ \\
\hline OH/ECW, \% & $0.04 \pm 6.47$ & $13.16 \pm 3.74$ & $31.86 \pm 8.68$ & $<0.001$ & $<0.001$ \\
\hline ECW/TBW & $0.46 \pm 0.03$ & $0.49 \pm 0.03$ & $0.54 \pm 0.03$ & $<0.001$ & $<0.001$ \\
\hline
\end{tabular}

Table 2. Comparison of demographics, serum chemistry, echocardiographic findings, and volume status according to OH/ECW tertiles. OH/ECW tertiles 1, 2, and 3 correspond to $<6.93,6.93-19.66$, and $>19.66 \%$, respectively. $B M I$ body mass index, $D B P$ diastolic blood pressure, $E C W$ extracellular water, $e G F R$ estimated glomerular filtration rate, $H D L-C$ high-density lipoprotein cholesterol, $h s$ - $C R P$ high-sensitivity C-reactive protein, $i P T H$ intact parathyroid hormone, $L A D$ left atrial dimension, $L A V I$ left atrial volume index, $L D L-C$ low-density lipoprotein cholesterol, $L V E D D$ left ventricular end-diastolic dimension, $L V E F$ left ventricular ejection fraction, $L V E D V$ left ventricular end-diastolic volume, $L V M I$ left ventricular mass index, NT-proBNP $\mathrm{N}$-terminal prohormone of B-type natriuretic peptide, $\mathrm{OH}$ overhydration, $\mathrm{RAASi}$ Renin-angiotensinaldosterone system inhibitors, $R W T$ relative wall thickness, $S B P$ systolic blood pressure, $T B W$ total body water. ${ }^{\#} P$ values obtained by the linear-by-linear association method or Jonckheere-Terpstra test. ${ }^{\text {a}}$ Kruskal-Wallis test; Median (interquartile range). 
A

$\mathrm{P}<0.001 ; \mathrm{P}$ for trend $<0.001$

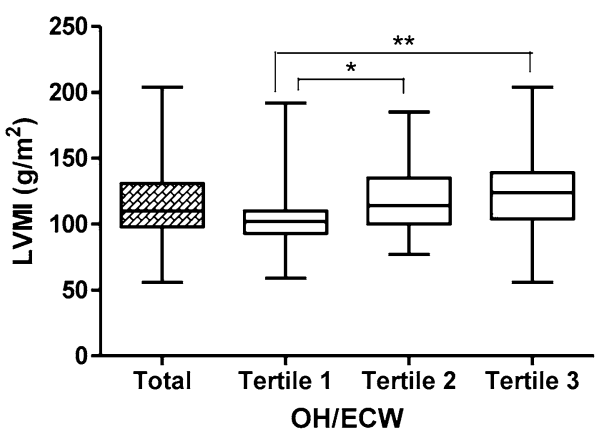

C

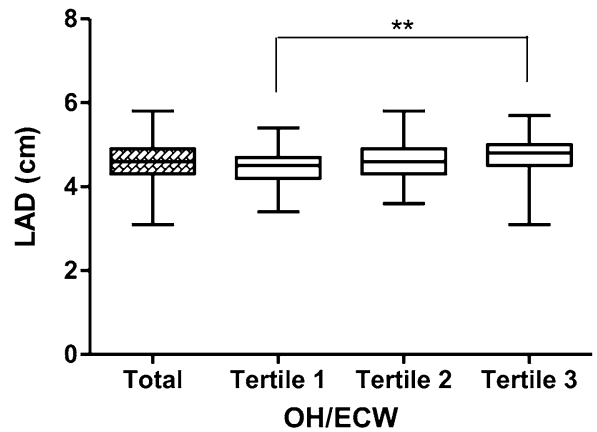

E

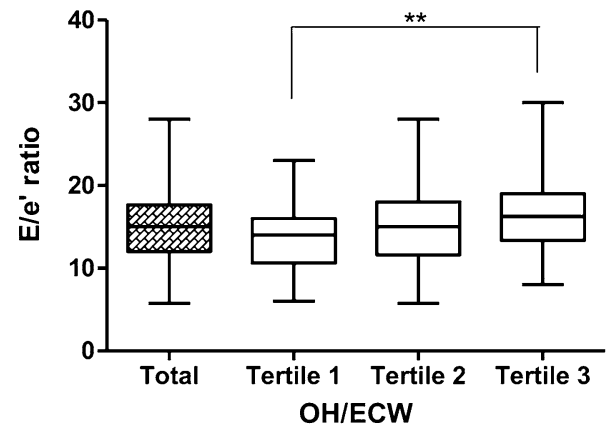

G

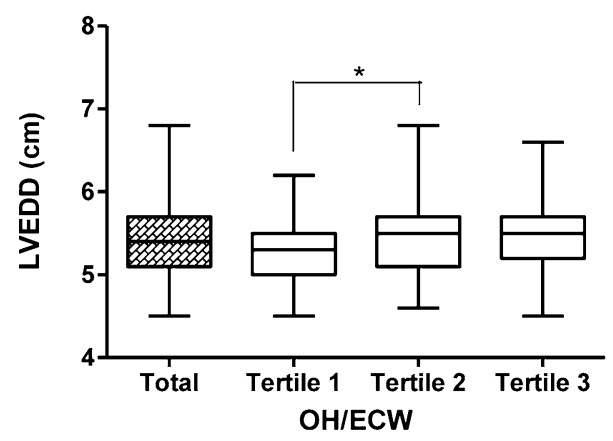

B

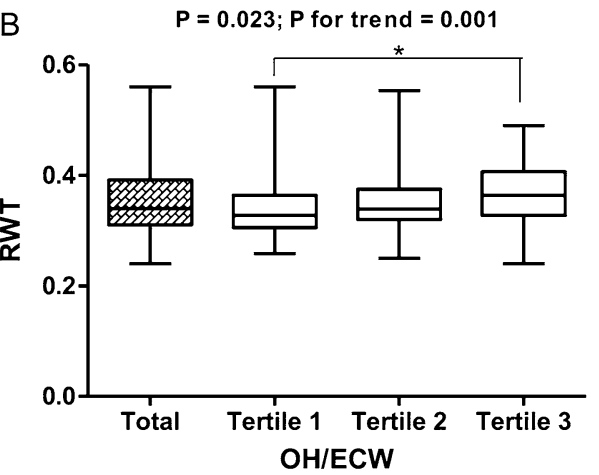

D $\quad P<0.001 ; P$ for trend $<0.001$

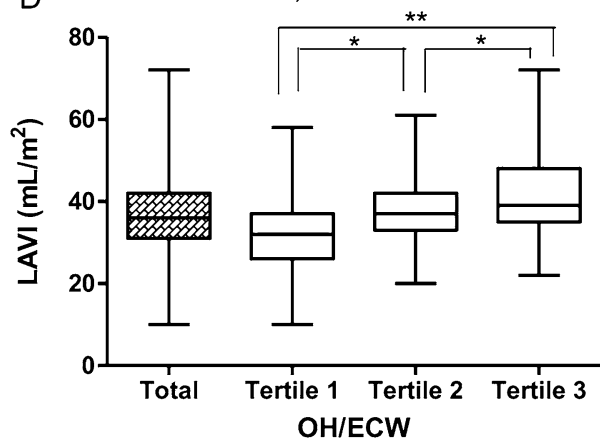

F

$P=0.552 ; P$ for trend $=0.933$

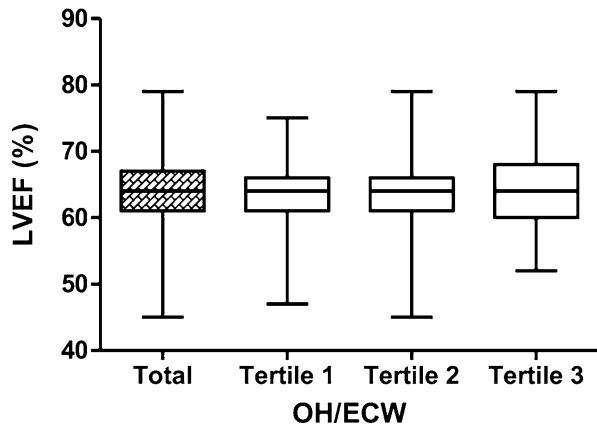

$\mathrm{H}$

$$
P=0.020 ; P \text { for trend }=0.034
$$

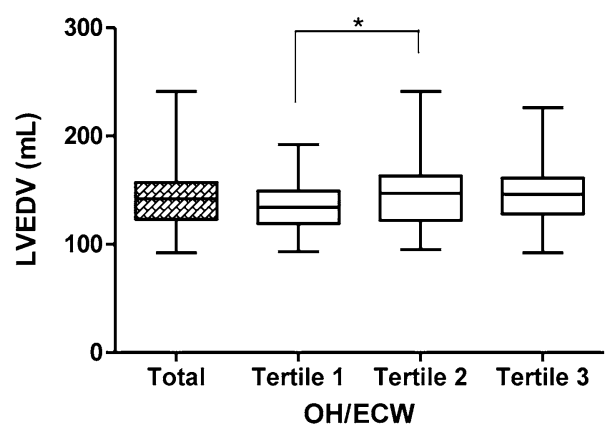

Figure 2. Left ventricular structural and functional alterations according to the distribution of $\mathrm{OH} / \mathrm{ECW}$ tertiles. OH/ECW tertiles 1,2 , and 3 correspond to $<6.93,6.93-19.66$, and $>19.66 \%$, respectively. ${ }^{\star} P<0.05$; ${ }^{* \star} P<0.001$, as tested by one-way ANOVA with post hoc Bonferroni correction. $P$ values for trend are also given. $E C W$ extracellular water, $L A D$ left atrial dimension, $L A V I$ left atrial volume index, $L V E D D$ left ventricular enddiastolic dimension, $L V E D V$ left ventricular end-diastolic volume, $L V E F$ left ventricular ejection fraction, $L V M I$ left ventricular mass index, $\mathrm{OH}$ overhydration, $R W T$ relative wall thickness. 


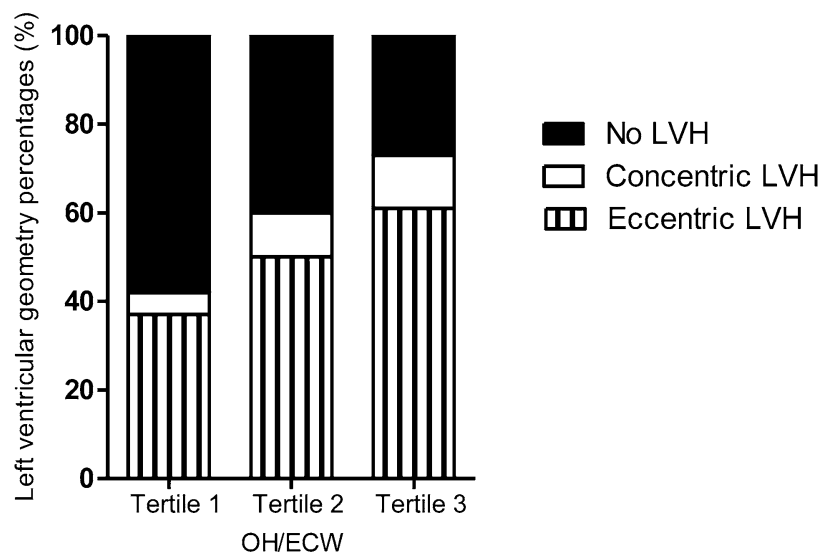

Figure 3. Percentage distribution of subjects according to the distribution of $\mathrm{OH} / \mathrm{ECW}$ tertiles. $\mathrm{OH} / \mathrm{ECW}$ tertiles 1,2 , and 3 correspond to $<6.93,6.93-19.66$, and $>19.66 \%$, respectively. $P$ for trend $<0.001$, as tested by the linear-by-linear association method. $E C W$ extracellular water, $L V H$ left ventricular hypertrophy, $\mathrm{OH}$ overhydration.

\begin{tabular}{|l|l|l|l|l|}
\hline \multirow{2}{*}{} & \multicolumn{2}{|l|}{ LVMI $^{\mathbf{a}}$} & \multicolumn{2}{l|}{ LVEDD $^{\mathbf{a}}$} \\
\cline { 2 - 5 } & B (95\% CI) & P value & B (95\% CI) & \multicolumn{1}{l|}{ P value } \\
\hline OH/ECW, \% & $0.321(0.069,0.573)$ & 0.013 & & $-0.272(-0.393,-0.151)$ \\
\hline Female (vs. male) & & & $0.035(0.021,0.050)$ & $<0.001$ \\
\hline BMI, kg/m ${ }^{2}$ & & & $0.004(0.001,0.007)$ & $<0.001$ \\
\hline SBP, mm Hg & $0.222(0.030,0.415)$ & 0.024 & $-0.064(-0.114,-0.015)$ & 0.012 \\
\hline Haemoglobin, g/dL & & & $-0.078(-0.137,-0.019)$ & 0.011 \\
\hline Calcium, mg/dL & & & & \\
\hline Phosphorus, $\mathrm{mg} / \mathrm{dL}$ & $3.550(1.249,5.852)$ & 0.003 & & \\
\hline
\end{tabular}

Table 3. Stepwise multiple linear regression of variables associated with left ventricular mass index and left ventricular end-diastolic dimension. Only significant determinants were presented. $B \beta$ coefficient, $B M I$ body mass index, $C I$ confidence interval, $D M$ diabetes mellitus, ECW extracellular water, $e G F R$ estimated glomerular filtration rate, $h s-C R P$ high-sensitivity C-reactive protein, $L V E D D$ left ventricular end-diastolic dimension,

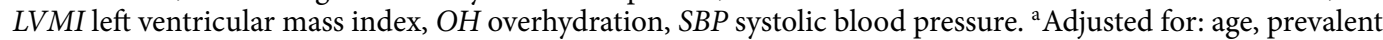
DM, diuretics use, hs-CRP, albumin, and eGFR.

When evaluating $\mathrm{OH} / \mathrm{ECW}$ as a continuous variable in a multivariate logistic regression analysis, $\mathrm{OH} / \mathrm{ECW}$ showed a substantial odds ratio in all three models. In addition, similar results were observed when OH/ECW was analysed in tertiles (Table 5). Serum phosphorus showed significant odds ratio in fully adjusted models, whereas the statistical significance of SBP disappeared in model 3. In all analytical models, the $P$ value of the Hosmer-Lemeshow test exceeded 0.05 .

Cut-off values of variables. Receiver operating characteristic (ROC) curves were drawn for OH, ECW/ TBW, serum phosphorus, and OH/ECW to determine the cut-off values for predicting LVH. The optimal OH/ ECW cut-off point for LVH was $10.050 \%$, with a sensitivity of $69.2 \%$ and a specificity of $58.3 \%$ (Table 6 ).

\section{Discussion}

Previous studies have illustrated that there are various clinical features of LV geometry in the CKD population ${ }^{20}$. An example is the wide heterogeneity in the prevalence of $\mathrm{LVH}$, which may be due to the differences in the number of enrolled patients, the proportion of subjects, the presence of comorbidities, and whether or not the patient is undergoing dialysis treatment. It is also important to note that the inspection tools and diagnostic criteria used to diagnose LVH were not the same in each study.

As the pathophysiology of LV remodelling is very complex, it may be difficult to generalise the evolution of $\mathrm{LVH}$ with only one associated factor in patients with CKD. Although blood pressure still plays a major role in inducing $\mathrm{LV}$ remodelling in $\mathrm{CKD}$, some other CKD-related factors are also known to be involved in this process. Some studies have proposed the independent risk factors for $\mathrm{LVH}$ in patients with $\mathrm{CKD} 5-\mathrm{ND}^{7}$, patients undergoing haemodialysis ${ }^{21}$, and patients undergoing peritoneal dialysis ${ }^{12}$. Chronic inflammation, anaemia, hypoalbuminaemia, hyperphosphataemia, arterial hypertension, and/or arterial stiffness, and high serum fibroblast 


\begin{tabular}{|l|l|l|l|l|}
\hline \multirow{2}{*}{ Variable } & Unadjusted & Model 1 & Model 2 & Model 3 \\
\cline { 2 - 5 } & B $(\mathbf{9 5 \%}$ CI $)$ & B (95\% CI) & B (95\% CI) & B (95\% CI) \\
\hline OH/ECW analysed as a continuous variable \\
\hline OH/ECW, \% & $0.360(0.124,0.596)$ & $0.384(0.145,0.623)$ & $0.475(0.219,0.731)$ & $0.381(0.029,0.732)$ \\
\hline SBP, mm Hg & $0.223(0.045,0.400)$ & $0.196(0.014,0.378)$ & $0.196(0.012,0.380)$ & $0.172(-0.035,0.379)$ \\
\hline Phosphorus, mg/dL & $3.192(1.007,5.376)$ & $3.373(1.174,5.572)$ & $2.808(0.568,5.048)$ & $2.270(-0.560,5.100)$ \\
\hline OH/ECW analysed in tertiles & \multicolumn{5}{|l|}{} \\
\hline OH/ECW, \% & Reference & Reference & Reference & Reference \\
\hline Tertile 1 & $7.132(-1.321,15.586)$ & $6.954(-1.605,15.514)$ & $8.525(-0.080,17.129)$ & $7.110(-2.794,17.013)$ \\
\hline Tertile 2 & $14.146(5.733,22.559)$ & $14.412(5.942,22.882)$ & $16.336(7.514,25.158)$ & $12.145(-0.180,24.470)$ \\
\hline Tertile 3 & $0.223(0.043,0.403)$ & $0.204(0.021,0.387)$ & $0.209(0.023,0.395)$ & $0.183(-0.025,0.391)$ \\
\hline SBP, mm Hg & $3.081(0.889,5.273)$ & $3.263(1.046,5.480)$ & $2.731(0.462,5.000)$ & $2.218(-0.641,5.077)$ \\
\hline Phosphorus, mg/dL
\end{tabular}

Table 4. Factors independently associated with left ventricular mass index. Model 1: Adjusted for age and sex. Model 2: Adjusted for age, sex, BMI, prevalent DM, and use of diuretics, renin-angiotensin-aldosterone system inhibitors, and phosphorous binders. Model 3: Adjusted for age, sex, BMI, prevalent DM, use of diuretics, renin-angiotensin-aldosterone system inhibitors, and phosphorous binders, haemoglobin, albumin, calcium, eGFR, and hs-CRP. OH/ECW tertiles 1, 2, and 3 correspond to $<6.93,6.93-19.66$, and $>19.66 \%$, respectively. $B \beta$ coefficient, $B M I$ body mass index, $C I$ confidence interval, DM diabetes mellitus, $E C W$ extracellular water, $e G F R$ estimated glomerular filtration rate, $h s$ - $C R P$ high-sensitivity C-reactive protein, $O H$ overhydration, SBP systolic blood pressure.

\begin{tabular}{|c|c|c|c|c|c|c|}
\hline \multirow[b]{2}{*}{ Variable } & \multicolumn{2}{|l|}{ Model 1} & \multicolumn{2}{|l|}{ Model 2} & \multicolumn{2}{|l|}{ Model 3} \\
\hline & OR $(95 \% \mathrm{CI})$ & $P$ value & OR (95\% CI) & $P$ value & OR $(95 \% \mathrm{CI})$ & $P$ value \\
\hline \multicolumn{7}{|c|}{$\mathrm{OH} / \mathrm{ECW}$ analysed as a continuous variable } \\
\hline $\mathrm{OH} / \mathrm{ECW}, \%$ & $1.039(1.013-1.065)$ & 0.003 & $1.043(1.015-1.071)$ & 0.003 & $1.042(1.008-1.076)$ & 0.014 \\
\hline SBP, $\mathrm{mm} \mathrm{Hg}$ & $1.022(1.004-1.041)$ & 0.017 & $1.023(1.004-1.042)$ & 0.018 & $1.019(0.998-1.040)$ & 0.077 \\
\hline Phosphorus, mg/dL & $1.413(1.121-1.781)$ & 0.003 & $1.423(1.123-1.803)$ & 0.003 & $1.482(1.098-1.999)$ & 0.010 \\
\hline \multicolumn{7}{|c|}{$\mathrm{OH} / \mathrm{ECW}$ analysed in tertiles } \\
\hline \multicolumn{7}{|c|}{$\mathrm{OH} / \mathrm{ECW}, \%$} \\
\hline Tertile 1 & \multicolumn{2}{|l|}{ Reference } & \multicolumn{2}{|l|}{ Reference } & \multicolumn{2}{|l|}{ Reference } \\
\hline Tertile 2 & $2.083(0.900-4.823)$ & 0.087 & $2.088(0.894-4.875)$ & 0.089 & $2.262(0.870-5.883)$ & 0.094 \\
\hline Tertile 3 & $3.839(1.619-9.105)$ & 0.002 & $4.122(1.664-10.211)$ & 0.002 & $3.907(1.336-11.430)$ & 0.013 \\
\hline SBP, $\mathrm{mm} \mathrm{Hg}$ & $1.023(1.004-1.042)$ & 0.015 & $1.024(1.005-1.043)$ & 0.015 & $1.020(0.998-1.041)$ & 0.072 \\
\hline Phosphorus, mg/dL & $1.396(1.106-1.763)$ & 0.005 & $1.413(1.113-1.794)$ & 0.005 & $1.469(1.087-1.985)$ & 0.012 \\
\hline
\end{tabular}

Table 5. Multivariate analysis using logistic regression for predictive factors of left ventricular hypertrophy. Model 1: Adjusted for age and sex. Model 2: Adjusted for age, sex, BMI, prevalent DM, and use of diuretics, renin-angiotensin-aldosterone system inhibitors, and phosphorous binders. Model 3: Adjusted for age, sex, $\mathrm{BMI}$, prevalent DM, use of diuretics, renin-angiotensin-aldosterone system inhibitors, and phosphorous binders, haemoglobin, calcium, eGFR, and hs-CRP. OH/ECW tertiles 1, 2, and 3 correspond to $<6.93$, 6.93-19.66, and $>19.66 \%$, respectively. CI confidence interval, $B M I$ body mass index, DM diabetes mellitus, $E C W$ extracellular water, $e G F R$ estimated glomerular filtration rate, $h s$-CRP high-sensitivity C-reactive protein, $\mathrm{OH}$ overhydration, $\mathrm{OR}$ odds ratio, $S B P$ systolic blood pressure.

\begin{tabular}{|l|l|l|l|l|l|l|}
\hline & Cut-off point & AUC & SE & Sensitivity & Specificity & $P$ value \\
\hline OH, litre & 1.550 & 0.636 & 0.040 & $69.2(\%)$ & $56.0(\%)$ & 0.001 \\
\hline OH/ECW, \% & 10.050 & 0.646 & 0.039 & $69.2(\%)$ & $58.3(\%)$ & $<0.001$ \\
\hline ECW/TBW & 0.469 & 0.648 & 0.039 & $64.1(\%)$ & $65.5(\%)$ & $<0.001$ \\
\hline Phosphorus, mg/dL & 5.400 & 0.657 & 0.039 & $72.6(\%)$ & $56.0(\%)$ & $<0.001$ \\
\hline
\end{tabular}

Table 6. Cut-off values of volume markers and serum phosphorus for left ventricular hypertrophy. AUC area under curve, $E C W$ extracellular water, $O H$ overhydration, $S E$ standard error, $T B W$ total body water.

growth factor 23 (FGF-23) levels were suggested as contributing factors for LVH in patients with CKD not 
undergoing dialysis ${ }^{22-25}$.

In the present study, the levels of hs-CRP, an inflammatory marker, tended to increase with increasing volume ( $P$ for trend $<0.001$ ); however, there was no significant difference between the patients with or without LVH $(P=0.513)$ and there was no association with LVMI. In stepwise multiple linear regressions, haemoglobin and serum calcium levels were associated with LVEDD, but not with LVMI. Sex differences and the contribution of BMI to LVH development were not significant in our study (Table 2, Supplementary Table S1). The prevalence of $\mathrm{LVH}$ in the general population with diabetes is known to be $\mathrm{high}^{26}$, but there was no difference in the prevalence of LVH between patients with and without diabetes in our study (Table 1). This is probably due to a combination of factors other than diabetes, such as chronic inflammation, hypertension, anaemia, and fluid overload.

Increased serum phosphorus levels have been associated with an increased risk of cardiovascular mortality in patients with CKD stages 3-4 ${ }^{27}$. Serum phosphorus is independently associated with LV mass in patients with $\mathrm{CKD}$. The reasons for this association are unclear. However, previous studies had suggested possible causes such as vascular calcification, arterial stiffness, and increased ventricular workload in higher serum phosphorus ${ }^{24,28}$. In a multivariate logistic regression analysis, serum phosphorus levels were found to be substantially associated with LVH. This suggests that lowering phosphorus levels can be considered as one of the therapeutic goals toward improving cardiovascular outcomes associated with LVH from an early stage of CKD.

Arterial hypertension is independently associated with $\mathrm{LVH}$ in patients with $\mathrm{CKD}^{1}$. Although hypertension is not a reliable biomarker of volume overload in patients with ESRD, the effect of volume overload on blood pressure is crucial. LVH represents an adaptive response to volume and/or pressure overload ${ }^{2}$. It is well known that cLVH results from increased afterload such as hypertension, whereas eLVH is a consequence of increased preload such as fluid overload and/or renal anaemia ${ }^{29}$. We do not know exactly whether fluid overload directly or indirectly influences left ventricular remodeling in this study. Considering the high eLVH patient ratio in our study, it suggests that there is a limit to explaining that the development of LVH in patients with advanced CKD is simply caused by hypertension.

Fluid overload has been associated with cardiovascular morbidity and all-cause mortality in patients with CKD not undergoing dialysis ${ }^{17,30,31}$. The poor prognosis of fluid overload is mainly explained by the link with cardiovascular effects such as LVH, LV systolic and diastolic dysfunction, pulmonary hypertension, and increased aortic stiffness ${ }^{32}$. The relationship between fluid imbalance and LV remodelling has been primarily elucidated through studies involving patients on dialysis ${ }^{11,12,19}$. The association of fluid overload and cardiac dysfunction has been rarely reported in patients with advanced $\mathrm{CKD}^{33,34}$. Moreover, few studies have directly and objectively measured volume status and selectively compared volume status with $\mathrm{LVH}^{18,35}$. Volume status assessed by bioimpedance spectroscopy (BIS) was not independently associated with LV mass measured by magnetic resonance imaging in patients with mild-to-moderate $\mathrm{CKD}^{5}$. $\mathrm{LVH}$ was not associated with relative fluid overload in patients undergoing haemodialysis ${ }^{36}$. However, relative overhydration was independently associated with LVH in multivariate analyses in our study. The differences from the results of previously mentioned studies may be due to differences in the enrolment criteria, patient cohort composition, and diagnostic criteria for the analysis. NT-proBNP, a marker for fluid overload in our study, showed significantly different levels between patients with and without LVH $(P<0.001)$. NT-proBNP is known to be associated with $\mathrm{LVH}^{37}$. However, it is unclear whether NT-proBNP itself reflects volume status or whether it is a by-product of structural damage to the myocardium due to fluid overload.

This study includes several limitations. We did not perform stress echocardiography to exclude asymptomatic patients with underlying cardiac impairment such as ischaemic heart disease. We did not measure LV mass by cardiac magnetic resonance imaging. Although cardiac magnetic resonance imaging is the best way to define LV mass and patterns of $\mathrm{LVH}^{29}$, it is not used in routine practice in our hospital because it is more expensive than echocardiography and includes a risk for nephrogenic systemic fibrosis. Since our assessment was only performed at one point, we could not determine fluctuations in LV geometr ${ }^{38}$. Serial changes in cardiac structures related to the removal of overload fluid by renal replacement therapy could not be assessed over time. Therefore, the relationship between fluid overload and LVH does not allow us to evaluate causality in this observational study. In addition, although the degree of urine volume and proteinuria were factors that could affect our research, we could not use them for analysis because they were not perfectly measured in all patients. However, this study has some strengths. First, all patients were free of intrinsic heart disease and represented a relatively homogenous population in terms of eGFR. Second, prior to performing dialysis treatments that may affect fluid balance, volume status was objectively measured at the time of echocardiography.

Our study demonstrates the specific levels of overhydration and serum phosphorus that affect the development of LVH in patients with CKD5-ND. Regardless of CKD stage, we can effectively prevent LV remodelling and improve outcomes through the tailored application of treatment strategies aimed at the risk factors identified in this study. Therefore, our results suggest that structural and functional evaluation of the heart using echocardiography and volume status assessment using bioimpedance should be performed simultaneously in patients with early-stage CKD rather than only in patients with stage $5 \mathrm{CKD}$, regardless of evident cardiovascular disease. Further research is needed to validate the consistency of this association across other stages of CKD.

\section{Materials and methods}

Patients and data collection. From September 2014 to December 2019, we have registered consecutive patients with CKD5 to a bioimpedance cohort. Therefore, the current study was a retrospective observational analysis of a prospective cohort database. All patients were hospitalised to plan their first dialysis treatment. Patients registered to the cohort underwent BIS, echocardiography, and laboratory evaluation at the time of enrolment, prior to dialysis. 
We excluded 51 patients with structural and functional cardiac abnormalities to reduce the effects of underlying heart disease that could cause LVH. We also excluded 3 patients with liver cirrhosis to minimise the impact of fluid imbalance on the study results. Patients who had a history of angina or myocardial infarction and patients who had findings of infarction on electrocardiography or had regional wall motion abnormalities on echocardiographic examination were considered as patients with coronary artery disease. Patients with concentric remodelling were excluded from the analysis because the total number of these patients was too small for analysis $(n=4)$ (Fig. 1).

This study was conducted in accordance with the Declaration of Helsinki. This study was initiated after receiving approval (no. CR316024) from the Institutional Review Board of Yonsei University Wonju Severance Christian Hospital. All patients provided written inform consent prior to participation in the study.

Conventional echocardiographic study. Echocardiography was performed in the harmonic imaging mode using a 3-MHz transducer and commercial ultrasound system (GE Vivid E9; GE Healthcare, Chicago, IL USA). The LV mass was calculated following the American Society of Echocardiography recommendations using the following equation:

$$
\mathrm{LV} \text { mass }=0.8 \times\left\{1.04 \times\left([\mathrm{PWTd}+\mathrm{SWTd}+\mathrm{LVDd}]^{3} \times[\mathrm{LVEDD}]^{3}\right)\right\}+0.6 \mathrm{~g}
$$

where PWTd and SWTd are the posterior and septal wall thickness at end-diastole, respectively, and the LVEDD is the M-mode LV dimension with the short axis view at end-diastole. To correct for body surface area, the LVMI was calculated by dividing LV mass by body surface area (BSA), using the formula as follows: BSA $=(0.00718$ $4 \times$ weight $^{0.425} \times$ height $\left.^{0.725}\right) \mathrm{m}^{2}$. The LA volume can be computed by using the area-length approximation: LA volume $=[8 /(3 \pi)][(\mathrm{A} 1 \times \mathrm{A} 2) / \mathrm{L}]$, where $\mathrm{A} 1$ and $\mathrm{A} 2$ are the corresponding LA areas measured in the apical twoand four-chamber views. The LA length $L$ is defined as the shortest of the two long axes measured in each view. The LA volume index (LAVI) was calculated by dividing LA volume by BSA. LVEDV and LVEF were measured using the biplane modified Simpson's rule, according to the previously mentioned recommendations. Transmitral early diastolic (E wave) velocities and the peak early $\left(\mathrm{e}^{\prime}\right)$ diastolic mitral annular velocities at the septal mitral annulus were measured. We calculated the $\mathrm{E} / \mathrm{e}^{\prime}$ ratio.

LVH was defined as LVMI $>95 \mathrm{~g} / \mathrm{m}^{2}$ in women and $>115 \mathrm{~g} / \mathrm{m}^{2}$ in men. RWT was calculated by the formula: RWT $=(2 \times \mathrm{PWT}) / \mathrm{LVEDD}$. Patients were divided into 4 categories according to the RWT and the presence of LVH as follows: normal geometry (no LVH, RWT $\leq 0.42$ ), concentric remodelling (no LVH, RWT $>0.42$ ), eLVH $(\mathrm{LVH}, \mathrm{RWT} \leq 0.42)$, and cLVH $(\mathrm{LVH}, \mathrm{RWT}>0.42)^{39}$. Echocardiography was performed by trained cardiologists who were completely blinded to patient information.

Assessment of the volume status. BIS was performed using the BCM (Body Composition Monitoring, Fresenius Medical Care AG \& Co., Bad Homburg vor der Höhe, Germany) at enrolment prior to any renal replacement therapy. The validity of BIS in the general and dialysis populations has been demonstrated in comparison to gold standard methods. ECW, intracellular water, and TBW were automatically provided by BCM using equations of Moissl et al..$^{40}$. OH level, OH/ECW, ECW/TBW, and NT-proBNP level are generally used as markers of fluid balance. Extracellular fluid overload, represented as $\mathrm{OH}$, can be calculated from the difference between the actually measured ECW and the normally expected ECW ${ }^{41}$. As measurement of relative overhydration, $\mathrm{OH} / \mathrm{ECW}$ was primarily used to determine volume status for our analysis.

Laboratory evaluations. All laboratory assessments were also performed at enrolment. Complete blood cell count was collected using a Sysmex XE-2100 (Sysmex, Kobe, Japan). Clinical chemistry test items including creatinine, albumin, calcium, phosphorus, hs-CRP, total cholesterol, high-density lipoprotein cholesterol, lowdensity lipoprotein cholesterol, and triglyceride were measured using a Cobas 8000 Modular Analyzer (Roche Diagnostics GmbH, Mannheim, Germany). The eGFR from serum creatinine was calculated using the Modification of Diet in Renal Disease formula. The NT-proBNP level was measured using the Modular Analytics E170 System (Roche Diagnostics GmbH, Mannheim, Germany). The analytical measuring range of NT-proBNP was from 5 to $35,000 \mathrm{pg} / \mathrm{mL}$. The hs-CRP level less than $0.3 \mathrm{~g} / \mathrm{dL}(3 \mathrm{~g} / \mathrm{L})$ is normal.

Statistical analysis. Categorical variables were reported as frequencies and percentages, and continuous variables were reported as means with standard deviations or medians with interquartile ranges, as appropriate. All patients were classified into two groups according to the absence or presence of LVH and were divided into three groups according to tertiles of $\mathrm{OH} / \mathrm{ECW}$ for analysis. Differences in clinical variables between the two groups were tested with two-sample $t$-tests and Mann-Whitney $U$ tests for continuous variables. The nominal variables were compared using a chi-squared test and Fisher's exact test as appropriate. Patient characteristics were also compared across tertiles of OH/ECW using a chi-squared test, analysis of variance (ANOVA) with post hoc Bonferroni correction, and Kruskal-Wallis test. A linear-by-linear association method and a Jonckheere-Terpstra trend test were used for analysing trends in the OH/ECW tertiles. LVEDD, LVEDV, RWT, and LVMI can affect the LV geometry pattern. Therefore, we first performed stepwise linear regression analyses to identify the potential determinants of LV structural indices (LVMI, LVEDD) adjusted for age, prevalent diabetes, diuretics use, hs-CRP, albumin, and eGFR. Parameters significantly associated with LVMI or LVEDD $(P<0.05)$ were used preferentially in the multivariate regression analyses. Uses of anti-hypertensive drugs and phosphorous binders were also included in the multivariate models because of their established relationship with LVH despite the lack of statistical significance in our results. The covariates that had statistical significance in the univariate analysis and needed to be clinically considered were simultaneously entered into the multi- 
variate models. We performed a multiple linear regression analysis to explore the association of LVMI with the identified determinants. Multivariate logistic regression models were used to examine the associations of $\mathrm{OH} /$ ECW, SBP, and serum phosphorus with LVH. The goodness of fit for model reliability was assessed using the Hosmer-Lemeshow test, whereby we considered a value of $P<0.05$ to indicate that the model had a poor fit. $\mathrm{OH} / \mathrm{ECW}$ was considered as both a continuous and categorical variable in the multivariate linear regression and multivariate logistic regression analyses. Finally, an ROC curve was created to establish cut-off values of $\mathrm{OH}$, ECW/TBW, serum phosphorus, and OH/ECW that discriminate between patients with LVH and those without LVH. All analyses were performed with IBM SPSS Statistics software (version 23.0; IBM Corporation, Armonk, NY, USA). Graphs were generated with Prism software (version 5.02; GraphPad Software, San Diego, CA, USA). Statistically significant differences were defined as those having $P$ values $<0.05$.

Received: 21 March 2020; Accepted: 9 September 2020

Published online: 02 October 2020

\section{References}

1. Paoletti, E., Bellino, D., Cassottana, P., Rolla, D. \& Cannella, G. Left ventricular hypertrophy in nondiabetic predialysis CKD. Am. J. Kidney Dis. 46, 320-327 (2005).

2. Nardi, E. et al. Left ventricular hypertrophy and geometry in hypertensive patients with chronic kidney disease. J. Hypertens. 27, 633-641 (2009).

3. Foley, R. N. et al. Clinical and echocardiographic disease in patients starting end-stage renal disease therapy. Kidney Int. 47, 186-192 (1995).

4. Levin, A. et al. Left ventricular mass index increase in early renal disease: impact of decline in hemoglobin. Am. J. Kidney Dis. 34, 125-134 (1999).

5. Schneider, M. P. et al. Left ventricular structure in patients with mild-to-moderate CKD-a magnetic resonance imaging study. Kidney Int. Rep. 4, 267-274 (2018).

6. Park, M. et al. Associations between kidney function and subclinical cardiac abnormalities in CKD. J. Am. Soc. Nephrol. 23, 1725-1734 (2012).

7. Nitta, K. et al. Risk factors for increased left ventricular hypertrophy in patients with chronic kidney disease: Findings from the CKD-JAC study. Clin. Exp. Nephrol. 23, 85-98 (2019).

8. Cheng, L. T. et al. Sex difference in the prevalence of left ventricular hypertrophy in dialysis patients. Am. J. Nephrol. 29, 398-405 (2009).

9. Yilmaz, M. et al. The prevalence of hypertension, valve calcification and left ventricular hypertrophy and geometry in peritoneal dialysis patients. Kidney Blood Press. Res. 35, 431-437 (2012).

10. de Roij van Zuijdewijn, C. L. et al. Eccentric left ventricular hypertrophy and sudden death in patients with end-stage kidney disease. Am. J. Nephrol. 42, 126-133 (2015).

11. Fagugli, R. M. et al. Association between extracellular water, left ventricular mass and hypertension in haemodialysis patients. Nephrol Dial Transplant. 18, 2332-2338 (2003).

12. Cader, R. A., Ibrahim, O. A., Paul, S., Gafor, H. A. \& Mohd, R. Left ventricular hypertrophy and chronic fluid overload in peritoneal dialysis patients. Int. Urol. Nephrol. 46, 1209-1215 (2014).

13. Matsuo, H. et al. Echocardiographic assessment of cardiac structural and functional abnormalities in patients with end-stage renal disease receiving chronic hemodialysis. Circ. J. 82, 586-595 (2018).

14. Tsai, Y. C. et al. Association of fluid overload with cardiovascular morbidity and all-cause mortality in stages 4 and 5 CKD. Clin. J. Am. Soc. Nephrol. 10, 39-46 (2015).

15. Tripepi, G. et al. Prognostic values of left ventricular mass index in chronic kidney disease patients. Nephrol. Dial. Transplant. https ://doi.org/10.1093/ndt/gfz254 (2019).

16. Tripepi, G. et al. Reappraisal in two European cohorts of the prognostic power of left ventricular mass index in chronic kidney failure. Kidney Int. 91, 704-710 (2017).

17. Paoletti, E. et al. Associations of left ventricular hypertrophy and geometry with adverse outcomes in patients with CKD and hypertension. Clin. J. Am. Soc. Nephrol. 11, 271-279 (2016).

18. Essig, M. et al. Cardiovascular remodelling and extracellular fluid excess in early stages of chronic kidney disease. Nephrol. Dial. Transplant. 23, 239-248 (2008).

19. Juan-García, I. et al. Echocardiographic impact of hydration status in dialysis patients. Nefrologia 32, 94-102 (2012).

20. Di Lullo, L., Gorini, A., Russo, D., Santoboni, A. \& Ronco, C. Left ventricular hypertrophy in chronic kidney disease patients: from pathophysiology to treatment. Cardiorenal. Med. 5, 254-266 (2015).

21. Patel, R. K. et al. Determinants of left ventricular mass and hypertrophy in hemodialysis patients assessed by cardiac magnetic resonance imaging. Clin. J. Am. Soc. Nephrol. 4, 1477-1483 (2009).

22. Gupta, J. et al. Association between inflammation and cardiac geometry in chronic kidney disease: findings from the CRIC study. PLoS ONE 10, e0124772 (2015).

23. Stack, A. G. \& Saran, R. Clinical correlates and mortality impact of left ventricular hypertrophy among new ESRD patients in the United States. Am. J. Kidney Dis. 40, 1202-1210 (2002).

24. Zou, J. et al. Serum phosphorus is related to left ventricular remodeling independent of renal function in hospitalized patients with chronic kidney disease. Int. J. Cardiol. 221, 134-140 (2016).

25. Gutiérrez, O. M. et al. Fibroblast growth factor 23 and left ventricular hypertrophy in chronic kidney disease. Circulation 119, 2545-2552 (2009).

26. Dawson, A., Morris, A. D. \& Struthers, A. D. The epidemiology of left ventricular hypertrophy in type 2 diabetes mellitus. Diabetologia 48, 1971-1979 (2005).

27. Eddington, H. et al. Serum phosphate and mortality in patients with chronic kidney disease. Clin. J. Am. Soc. Nephrol. 5, 2251-2257 (2010).

28. Chue, C. D. et al. Serum phosphate is associated with left ventricular mass in patients with chronic kidney disease: a cardiac magnetic resonance study. Heart 98, 219-224 (2012).

29. Chiu, D. Y., Green, D., Abidin, N., Sinha, S. \& Kalra, P. A. Cardiac imaging in patients with chronic kidney disease. Nat. Rev. Nephrol. 11, 207-220 (2015).

30. Vega, A. et al. Any grade of relative overhydration is associated with long-term mortality in patients with Stages 4 and 5 non-dialysis chronic kidney disease. Clin. Kidney J. 11, 372-376 (2018). 
31. Faucon, A. L. et al. Extracellular fluid volume is associated with incident end-stage kidney disease and mortality in patients with chronic kidney disease. Kidney Int. 96, 1020-1029 (2019).

32. Kalantar-Zadeh, K. et al. Fluid retention is associated with cardiovascular mortality in patients undergoing long-term hemodialysis. Circulation 119, 671-679 (2009).

33. Yilmaz, A., Yilmaz, B., Küçükseymen, S., Özpelit, E. \& Pekel, N. Association of overhydration and cardiac dysfunction in patients have chronic kidney disease but not yet dialysis. Nephrol. Ther. 12, 94-97 (2016).

34. Kim, J. S., Yang, J. W., Yoo, J. S., Choi, S. O. \& Han, B. G. Association between E/e' ratio and fluid overload in patients with predialysis chronic kidney disease. PLoS ONE 12, e0184764 (2017)

35. Zoccali, C. \& Mallamaci, F. Mapping progress in reducing cardiovascular risk with kidney disease: managing volume overload. Clin. J. Am. Soc. Nephrol. 13, 1432-1434 (2018).

36. Cristina Di Gioia, M. et al. Echocardiographic findings in haemodialysis patients according to their state of hydration. Nefrologia 37, 47-53 (2017).

37. Mishra, R. K. et al. Association of N-terminal pro-B-type natriuretic peptide with left ventricular structure and function in chronic kidney disease (from the Chronic Renal Insufficiency Cohort [CRIC]). Am. J. Cardiol. 111, 432-438 (2013).

38. Eckardt, K. U. et al. Left ventricular geometry predicts cardiovascular outcomes associated with anemia correction in CKD. J. Am. Soc. Nephrol. 20, 2651-2660 (2009).

39. Lang, R. M. et al. Recommendations for cardiac chamber quantification by echocardiography in adults: an update from the American Society of Echocardiography and the European Association of Cardiovascular Imaging. J. Am. Soc. Echocardiogr. 28, 1-39.e14 (2015).

40. Moissl, U. M. et al. Body fluid volume determination via body composition spectroscopy in health and disease. Physiol. Meas. 27, 921-933 (2006).

41. Wabel, P., Chamney, P., Moissl, U. \& Jirka, T. Importance of whole-body bioimpedance spectroscopy for the management of fluid balance. Blood Purif. 27, 75-80 (2009).

\section{Author contributions}

Conceptualization, B.G.H; Methodology, B.G.H. and J.Y.L.; Software, S.O.C. and J.Y.L.; Validation, B.G.H. and J.W.Y.; Formal Analysis, B.G.H. and J.W.Y.; Investigation, B.G.H. and J.W.Y.; Resources, B.G.H., J.W.Y. and J.S.K.; Data Curation, B.G.H. and J.Y.L.; Writing-Original Draft Preparation, B.G.H.; Writing-Review \& Editing, B.G.H. and J.S.K.; Visualization, B.G.H. and J.Y.L.; Supervision, J.S.K.; Project Administration, B.G.H. and J.S.K.

\section{Competing interests}

The authors declare no competing interests.

\section{Additional information}

Supplementary information is available for this paper at https://doi.org/10.1038/s41598-020-73038-8.

Correspondence and requests for materials should be addressed to J.-S.K.

Reprints and permissions information is available at www.nature.com/reprints.

Publisher's note Springer Nature remains neutral with regard to jurisdictional claims in published maps and institutional affiliations.

(c) (i) Open Access This article is licensed under a Creative Commons Attribution 4.0 International License, which permits use, sharing, adaptation, distribution and reproduction in any medium or format, as long as you give appropriate credit to the original author(s) and the source, provide a link to the Creative Commons licence, and indicate if changes were made. The images or other third party material in this article are included in the article's Creative Commons licence, unless indicated otherwise in a credit line to the material. If material is not included in the article's Creative Commons licence and your intended use is not permitted by statutory regulation or exceeds the permitted use, you will need to obtain permission directly from the copyright holder. To view a copy of this licence, visit http://creativecommons.org/licenses/by/4.0/.

(C) The Author(s) 2020 